\title{
Shedding light on dark spots using EIS
}

\author{
Stig Ollmar \\ CLINTEC, Karolinska Institutet, Sweden \\ SciBase AB, Sweden \\ President ISEBI
}

My route into the exciting world of electrical bio-impedance started in the late 70's by not so bio things with applied physics and sensing technologies. My route to the physics department was not straight either, I actually planned to become a theoretical philosopher with a focus on the philosophy of space and time and cosmology. However, my knowledge of physics was found insufficient and during repair of this defect, I fell in love with laboratory work and simply hanged in there during a total thinking makeover, including self-organizing systems and oscillatory behavior in chemistry (e.g. the Belousov-Zhabotinsky reaction). In the field of electrochemistry, lumped parameter models mainly consisting of RC-networks worked well and were well established by Ross McDonald and others - typically one clean dispersion for each mechanism with none or only a little overlap. After that, the step was not too big to immensely more complex biological systems and how to measure on them - I thought.

I was more or less kidnapped by the Karolinska Institute who wanted a crazy physicist to do measurements on cats and patients. In the beginning mainly to characterize hard tissues like teeth, then oral mucosa, and soon enough also skin. For reasonably homogeneous tissues, lumped parameter models in the spirit of the Cole brothers were acceptable. But, skin is far from homogeneous and exhibits properties that would confound each other if modelled in such a simplistic way. My team found that the so called $\alpha$ dispersion was much bigger than, and to a large extent was overlapping the $\beta$ dispersion (as seen in a Nyqvist plot), which means that Cole-modelling would easily wipe out any trace of the $\beta$ dispersion although some funny little distortion of the semicircle did show up - but was first disregarded as imperfections in the measurement system. When we realized the information we wanted was hiding in the almost drowned $\beta$ dispersion, we tried simple indices and found that we could discriminate between different toxically elicited reactions using EIS, and correlate information in such spectra to the "gold standard" histopathology, we thought: Wow, maybe EIS could work also in the clinic as a decision support tool for medical doctors.

We decided to go for skin cancer, and particularly malignant melanoma which is a very dangerous disease, so dangerous that about 97 percent of all suspect lesions are normally treated as if they are malignant melanomas and therefore excised - just in case - and then found out to be harmless. We soon found out that information related to malignant melanoma was spread over a wide range of frequencies, i.e. the malignancy modulated the shape of the spectrum, and the spectrum thus contained more information than could be found by simple Cole-modelling or simple indices. Multivariate methods, such as PCA, give superior results and also quantifies how much of the information inherent in a spectrum is explained by each PC.

Elaborating such methods, and including a vast variety of malignant melanomas in various stages of development (all verified by a board of histopathologists) it was possible to achieve more than 97 percent sensitivity (meaning essentially no melanoma was missed) and, as reported in a large multicenter study, the largest of its kind ever, at least 34 percent specificity (meaning a significant reduction of surgical interventions compared to the common practice of cutting almost anything suspicious, thus close to zero specificity). A diagnostic decision support tool making medical doctors better doctors, by using an elaborated form of EIS. Work to enhance both sensitivity and in particular specificity further is in progress. 
To close the circle, back to philosophy. What are we really measuring? Has anyone seen an ohm? Many publications include lumped parameter models, networks of resistors and capacitors, but I have never seen neither a resistor nor a capacitor in a tissue while looking in the microscope, not even in the electron microscope. Remembering Niels Bohr and his philosophical thinkings: we cannot measure anything without influencing the object. So how do we know that the illusive ohm is there when we are not looking? Rubbish, someone might argue, it's only a relation between volts and amperes. But, did anyone actually see a volt or an ampere? The most real Ohm I can see in my imagination is the guy to the right, who established a sound physical foundation and

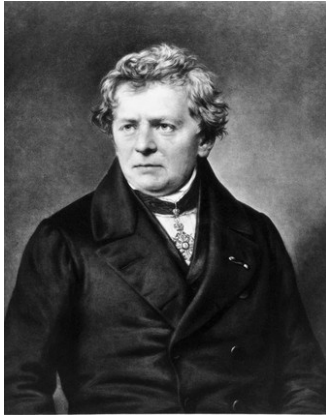

Georg Simon Ohm 1787- 1854 pointed out a path of discovery which is still unveiling its secrets, including those of living tissues. 